\title{
IDENTIFICATION AND SEGMENTATION OF TOUCHING BRAHMI CHARACTERS FROM DEGRADED DIGITAL ESTAMPAGE IMAGES USING ENSEMBLE CLASSIFIER
}

\author{
Aniket S. Nagane \\ Assistant Professor, MAEER's MIT-Arts, Commerce and Science College, Alandi, Pune 412105, India \\ Department of Computer Science, Savitribai Phule Pune University, Pune 411007, India; \\ asnagane@mitacsc.ac.in; aniket.nagane@gmail.com
}

Shankar M. Mali

Professor, Dr. Vishwanath Karad, MIT World Peace University, Pune India; Department of Computer Science, Savitribai Phule Pune University, Pune 411007, India shankar.mali@mitwpu.edu.in; shankarmali007@gmail.com

\begin{abstract}
Brahmi script is an ancient Indian script that requires expertise to read the written documents. OCR system can help reading and understanding the documents of Brahmi script. In the OCR systems, to recognize the symbols, accurate segmentation in earlier phase is required. If the segmentation goes wrong, it indicates the failure of the recognition process. Identification and segmentation of touching characters is a challenging task in OCR systems. In this paper, the authors have proposed an algorithm which successfully identifies and segments the touching characters from the Brahmi script documents. The algorithm uses ensemble classification technique to identify the touching characters using 9 unique features. The proposed algorithm has achieved $100 \%$ accuracy in identification and $99.16 \%$ accuracy in segmentation of touching Brahmi script characters. The results of proposed algorithm are significant to identify and correctly segment the touching Brahmi script characters from degraded digital estampage images.
\end{abstract}

Keywords: Brahmi script; estampage; OCR; segmentation; touching characters.

\section{Introduction}

In traditional India, people used to believe more in verbal transfer of knowledge rather than the written communication. History of written communication in Indian culture is newer as compared to the classical ancient cultures of China and Japan (Ojha 1971). The Brahmi script is considered to be oldest Indian ancient script in Central Asia and Indian Subcontinent. Brahmi script originated in BC 400 and was practiced till the period of AD 500 (Salomon 1998).

Brahmi script was widely used during the period of emperor Ashoka from BC 268 to BC 232 when it spread across the Indian subcontinent and became popular. Brahmi script inscriptions are mostly found engraved on rocks, copper-plates (tampra-patra) and bhurja-patra (Fig. 1). 


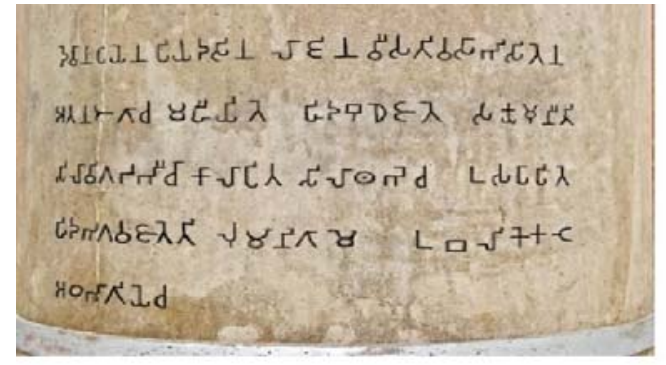

a

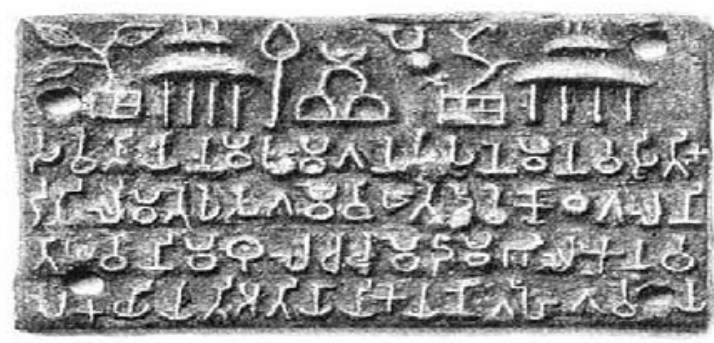

b

Fig. 1 Brahmi script inscriptions (a) rock inscriptions; (b) copper-plate (tamra-patra) inscription

Eventually, rock inscriptions of the Brahmi script are getting degraded day by day due to rock erosion. Archeological Survey of India (ASI), Mysore, India is the central government institute that preserves the inscriptions in the digital format by maintaining the glass negatives of estampages. Estampage is an impression of inscriptions on special paper (Fig. 2). The paper is inked from one side and is pasted on the inscription. Further, the impression is taken on the paper using slow wooden hammering (https://en.wikipedia.org/wiki/Estampage\#: :text=From\%20Wikipedia $\% 2 \mathrm{C} \% 20$ the $\% 20$ free $\% 20$ encyclopedia,pa per\%20for $\% 20 \mathrm{a} \% 20$ clearer $\% 20$ read [accessed on October 19, 2021]).

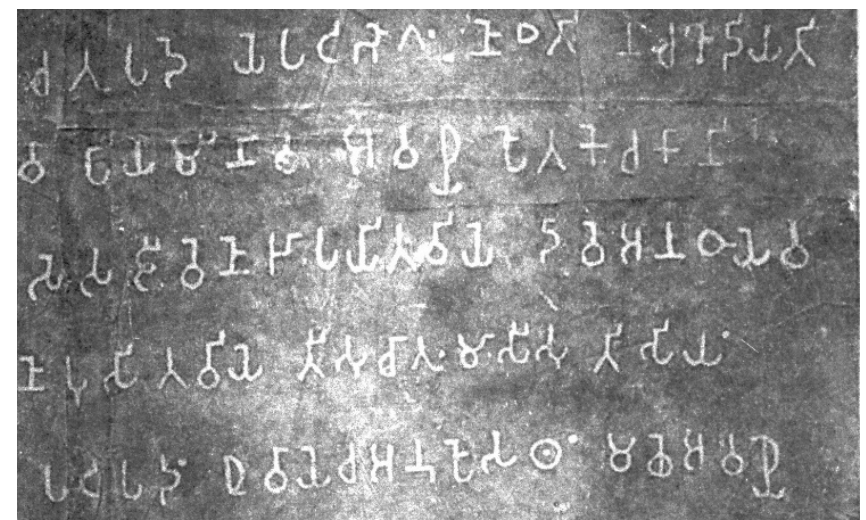

Fig. 2 Estampage of Brahmi script inscription (source: ASI, Mysore, India)

Considering the longer time span, diverse geographical areas, and usage of different mediums to write the Brahmi script, variations are observed in its appearance. The variations are found in the form of inconsistent character set, overlapping of text causing mixing of characters etc. Such Brahmi script documents are discovered even today. Only the experts of Brahmi script and contemporaneous languages can read such documents. On the other hand, optical character recognition (OCR), can definitely help transliterate the Brahmi script document into a document containing a modern-day script ( Garain et al. (2012); Jha and Parvathy (2019)). This will help anyone (those not having any expertise or knowledge about the Brahmi script) to read and understand the contents of the document

OCR systems are used to digitalize the documents available in hard copy (Hamad and Kaya (2016); Ullah et al. (2019); Zho et al. (2016)). Hard copy documents can be categorized as (i) Historical Documents; (ii) Handwritten Documents; and (iii) Printed Documents. These documents may contain one or several scripts (multilingual document), special symbols, images, or icons. OCR system converts these documents into a digitalized document with same contents (Karthick et al. (2019)). OCR system recognizes the engraved, handwritten, or printed characters from scripts' character set and generates the equivalent characters in digitalized form. In OCR systems, some operations are performed in a sequence to get the results. Some important operations are (i) Image Acquisition; (ii) Pre-processing; (iii) Segmentation; (iv) Feature Extraction; and (v) Classification and Recognition (Farulla et al. (2017); Mittal and Garg (2020)). Image acquisition is the very first and basic step of OCR system, in which, using some source we can capture an image of the object, wherein OCR objects are generally the hard-copy documents. Once the image has been captured, the next process is noise removal, image cropping, enhancement of the image as per the requirement of the further steps, etc. After this, the crucial step is segmentation of characters or symbols from the document. This segmentation can be done in the following steps: (i) Line Segmentation; (ii) Word Segmentation; and (iii) Character segmentation (Ali and Suresha (2019); Garain and Chaudhuri (2002); Peng et al. (2017); Rakshit et al. (2018)). The process of segmentation is a major operation in the OCR systems, where the results of next steps such as feature extraction and recognition are totally based on the accuracy of segmentation. Detail analysis of 
segmentation, lists the problems that cause in-accurate segmentation (Burra et al. (2018); Deshmukh and Kolhe (2019); Jain (2017); Ramalingam and Bhojan (2018)). After the accurate segmentation, feature vector is generated for every individual character. Using this feature vector, correct labelling of the characters is performed and recognition phase is completed.

\section{Analysis of Segmentation}

Segmentation techniques are mainly based on connectivity analysis of the objects in the image. A unique connected object is considered as a single character. When the two pixels in a digital image are not connected with each other, they are considered to be a part of two different objects. The historical documents contain problems like bleed through effect, broken characters, overlapping characters, touching characters, etc. In case of two-sided documents, over the years, the ink from one side of the page is visible on the other side which gives the bleed through effect. Over a long period, the ink on the papers fade out or the degradation of the papers may lead to broken characters. Overlapping characters are recognized when the strokes of characters from two adjacent rows are mixed into each other. Touching characters are similar to the overlapping ones where the strokes of adjacent characters in the same row get mixed. Here, mixing of strokes means characters have connected pixels (Patil and Mali (2015); Rehman and Saba (2011); Roy et al. (2009); Saba et al. (2011); Saba et al. (2014); Sen et al. (2018)).

In all the above cases, connectivity analysis-based segmentation does not work appropriately. In case of the bleed through effect, unnecessary shapes connect to the objects. In case of broken characters, single object gets segmented as two or more number of objects, and in case of overlapping and touching characters, two or more characters get segmented as one single character. In this paper, we have proposed the method to segment the touching characters of Brahmi script.

\section{Data Collection}

To implement the proposed method and test the results, we have created our image set consisting 8014 characters as no standard image set is available for Brahmi script characters. We have acquired all the images from Archeological Survey of India (ASI), Mysore, India and Bhandarkar Oriental Research Institute, Pune. By using these images, we have performed pre-processing and segmentation and have generated an image set of 8014 characters, including the touching characters. The same image set is used for identifying and segmenting the touching characters.

\section{Proposed Methodology}

The proposed method is implemented in the following parts: identification of touching characters and identification of segmentation points. In the first part of the algorithm, we identify touching and non-touching characters using ensembled classifier. Second part of the algorithm covers segmentation of touching characters using size of the characters and their aspect ratio with each other.

\subsection{Identification of touching characters using ensemble classifier}

Four different classification techniques viz. Ensemble classification using boosting algorithm, Support Vector Machine with Error correcting output codes, K-Nearest Neighbour and K-means clustering are implemented on the dataset. Ensemble classifier has produced better results comparatively, i.e., identification of touching characters is done using ensembled classification algorithm. Ensemble classification is a process where outcomes of all the present models are analysed and combined together to form a new model so as to acquire better results as compared to the individual models. While using individual classification models, some of the features from the feature set may not contribute to the final result, and hence, implementing the ensemble classification technique makes the use of all the features to improve the accuracy (Liu and Zhang (2019)).

Ensemble Classifier uses boosting algorithms for classification. AdaBoostM1 is a boosting algorithm for binary classification and AdaBoostM2 is an extension of AdaBoostM1 for multiple classes. The boosting algorithm trains learners sequentially. For every learner with index $t$, AdaBoostM1 computes the weighted classification error where as AdaBoostM2 uses weighted pseudo-loss for $N$ observations and $K$ classes

$$
\varepsilon_{t}=\frac{1}{2} \sum_{n=1}^{N} \sum_{k \neq y_{n}} d_{n, k}^{(t)}\left(1-h_{t}\left(x_{n}, y_{n}\right)+h_{t}\left(x_{n}, k\right)\right)
$$

where

- $h_{t}\left(x_{n}, y_{n}\right)$ is the confidence of prediction by learner at step $t$ into class $k$ ranging from 0 (not at all confident) to 1 (highly confident).

- $d_{n, k}^{(t)}$ are observation weights at step $t$ for class $k$.

- $y_{n}$ is the true class label taking one of the $K$ values. 
- The second sum is over all classes other than the true class $y_{n}$.

The default learning rate for boosting algorithms is 1 . When the learning rate of an algorithm is a lower number, its learning rate is also slow, however, when the learning rate is slow, it converges a better solution. Learning at a rate less than 1 is often called "shrinkage".

In AdaBoostM2, classification accuracy is measured as Pseudo-loss. Pseudo-loss behaviour can be explained as the first few learners in a boosted ensemble give low pseudo-loss values. After the first few training steps, the ensemble begins to learn at a slower pace, and the pseudo-loss value approaches 0.5 from below.

We also have implemented individual classification models which are KNN, SVM, and K-means clustering.

- K-nearest neighbour is supervised machine-learning algorithm used for classification. The behaviour of this classification model when the dataset or weights contain missing observations can be described as below:

If any value of class labels $(\mathrm{Y})$ or any weight is missing, then it removes those values from class labels, the weights, and the corresponding rows of predictor data(x) from the data. The software renormalizes the weights to sum to 1 .

When standardized predictors are specified without scale, classification model removes missing observations from individual predictors before computing the mean and standard deviation.

When standardized predictor is specified with Prior or Weights, then the observations weights are also considered. Specifically, the weighted mean of predictor $j$ is

$$
\overline{X_{j}}=\sum_{B_{i}} w_{k} X_{j k}
$$

and the weighted standard deviation is

$$
s_{j}=\sum_{B_{i}} w_{k}\left(x_{j k}-\overline{x_{j}}\right)
$$

where $B_{j}$ is the set of indices $k$ for which $x_{j k}$ and $w_{k}$ are not missing.

When the Mahalanobis distance is specified without scale or covariance matrix, classification model removes rows of $X$ that contain at least one missing value. In this case, the model does following things:

(1) Computes the means and standard deviations of each predictor

(2) Standardizes the data using the results of step 1

(3) Computes the distance parameter values using their respective default.

If you specify Scale and either of Prior or Weights, then the software scales observed distances by the weighted standard deviations.

If you specify Cov and either of Prior or Weights, then the software applies the weighted covariance matrix to the distances. In other words,

$$
\operatorname{Cov}=\frac{\sum_{B} w_{j}}{\left(\sum_{B} w_{j}\right)^{2}-\sum_{B} w_{j}^{2}} \sum_{B} w_{j}\left(x_{j}-\bar{x}\right)^{\prime}\left(x_{j}-\bar{x}\right)
$$

where $B$ is the set of indices $j$ for which the observation $x_{j}$ does not have any missing values and $w_{j}$ is not missing.

- Support Vector Machines (SVMs) are a set of supervised learning methods. In general, SVMs are used for binary classification problems. By using Error-Correcting Output Codes Technique, we can redesign multiclass classification problem as multiple binary classification problems. We have used SVM with ECOC and its model can be designed as below:

Let $M$ be the coding design matrix with elements $m_{k l}$, and $s_{l}$ be the predicted classification score for the positive class of learner $l$. The algorithm assigns a new observation to the class ( $\left.{ }^{\wedge} k\right)$ that minimizes the aggregation of the losses for the $L$ binary learners. 


$$
\hat{k}=\underset{k}{\arg \min } \frac{\sum_{l=1}^{L}\left|m_{k l}\right| g\left(m_{k l}, s_{l}\right)}{\sum_{l=1}^{L}\left|m_{k l}\right|}
$$

ECOC models can improve classification accuracy, compared to other multiclass models.

- $\quad k$-Means Clustering is unsupervised machine learning algorithm used to group datapoints with similar properties. $k$-means clustering is an iterative, data-partitioning algorithm that assigns $n$ observations to exactly one of $k$ clusters defined by centroids, where $k$ is chosen before the algorithm starts.

The algorithm proceeds as follows:

(1) Choose $k$ initial cluster centers (centroid). For example, choose $k$ observations at random.

(2) Compute point-to-cluster-centroid distances of all observations to each centroid.

(3) Compute the average of the observations in each cluster to obtain $k$ new centroid locations.

(4) Repeat steps until cluster assignments do not change, or the maximum number of iterations is reached.

For classification, we have to identify unique features from the image. We have identified 9 such features to distinguish the image as touching or non-touching character (Fig. 3). All features are described below

(1) AF: It is a feature which describes the area of an object. Let I is an image defined as $f(i, j)$ and $J$ is an image which is a subset of I determined as 8 connected component from the image then number of pixels in $\mathrm{J}$ is indicates the area of $\mathrm{J}$

$\mathrm{AF}=|\mathrm{A}|$ where $\mathrm{A}$ is collection of pixels belonging to $\mathrm{J}$

(2) CAF: Convex area feature is a numeric value indicating the number of pixels in convex hull $\mathrm{C}_{\mathrm{h}}$ of the object.

$\mathrm{CAF}=|\mathrm{C}|$ where $\mathrm{C}$ is collection of pixels belonging to $\mathrm{C}_{h}$

(3) PF: Perimeter feature is the numeric value computed as number of pixels lying around the border B of object region.

$\mathrm{PF}=|\mathrm{P}|$ where $\mathrm{P}$ collection of pixels belonging to $\mathrm{B}$

(4) MAL: Major Axis length feature is a scalar value which describes number of pixels forming the major axis ' $M$ ' of the image ellipse.

$\mathrm{MAL}=|\mathrm{M}|$ where $\mathrm{M}$ is major axis of the image ellipse

(5) MnAL: Major Axis length feature is a scalar value which describes the number of pixels forming minor axis ' $\mathrm{Mn}$ ' of the image ellipse.

$\mathrm{MnAL}=|\mathrm{Mn}|$ Where $\mathrm{Mn}$ is minor axis of the image ellipse.

(6) Cnt1: It is the numeric value indicating number of spikes (high) in a vertical projection profile of first horizontal part of an image.

$\mathrm{Cnt} 1=|\mathrm{T}|$

$\mathrm{T}=\{\mathrm{x} / \mathrm{x} \in \mathrm{vp} \& \mathrm{x}<=1\}$

$\mathrm{Vp}=\{\mathrm{y} / \mathrm{y}=\mathrm{sm}\}$

$\operatorname{Sm}(\mathrm{i})=\sum_{r=1, c=i}^{n / 2} f(r, c)$

where $n$ is number of rows, $i=1$ to $m$ and $m$ is number of columns

(7) Cnt2: It is the numeric value indicating number of valleys (low) in a vertical projection profile of first horizontal part of an image

Cnt2 $=|\mathrm{T}|$

$\mathrm{T}=\{\mathrm{x} / \mathrm{x} \in \mathrm{vp} \& \mathrm{x}>1\}$

$\mathrm{Vp}=\{\mathrm{y} / \mathrm{y}=\mathrm{sm}\}$

$\operatorname{Sm}(\mathrm{i})=\sum_{r=1, c=i}^{n / 2} f(r, c)$

where $n$ is number of rows, $i=1$ to $m$ and $m$ is number of columns

(8) Cnt3: It is the numeric value indicating number of spikes (high) in a vertical projection profile of second horizontal part of an image.

$\mathrm{Cnt} 3=|\mathrm{T}|$

$\mathrm{T}=\{\mathrm{x} / \mathrm{x} \in \operatorname{vp} \& \mathrm{x}<=1\}$

$\mathrm{Vp}=\{\mathrm{y} / \mathrm{y}=\mathrm{sm}\}$ 
$\operatorname{Sm}(\mathrm{i})=\sum_{r=n / 2+1, c=i}^{n} f(r, c)$

where $n$ id number of rows, $i=1$ to $m$ and $m$ is number of columns

(9) Cnt4: It is the numeric value indicating number of valleys (low) in a vertical projection profile of second horizontal part of an image

$\mathrm{Cnt} 3=|\mathrm{T}|$

$\mathrm{T}=\{\mathrm{x} / \mathrm{x} \in \mathrm{vp} \& \mathrm{x}>1\}$

$\mathrm{Vp}=\{\mathrm{y} / \mathrm{y}=\mathrm{sm}\}$

$\operatorname{Sm}(\mathrm{i})=\sum_{r=n / 2+1, c=i}^{n} f(r, c)$

where $n$ id number of rows, $i=1$ to $m$ and $m$ is number of columns

Using these features, we implemented ensembled classification technique to identify touching characters.

Table 1 describes sample of features for touching and non-touching characters.

\begin{tabular}{|c|c|c|c|c|c|c|c|c|c|}
\hline Type of character & $\mathrm{AF}$ & $\mathrm{CAF}$ & MAL & MnAL & Peri & cnt1 & cnt2 & cnt3 & cnt4 \\
\hline \multirow{5}{*}{ Touching } & 381 & 1299 & 63.62592 & 44.46662 & 289.73 & 34 & 20 & 4 & 51 \\
\cline { 2 - 10 } & 417 & 1167 & 48.70971 & 43.76582 & 214.611 & 20 & 20 & 2 & 35 \\
\cline { 2 - 10 } & 467 & 1054 & 85.21813 & 37.86985 & 229.929 & 6 & 36 & 13 & 32 \\
\cline { 2 - 10 } & 402 & 1304 & 47.6636 & 37.52782 & 272.398 & 16 & 29 & 3 & 44 \\
\cline { 2 - 10 } & 432 & 1027 & 83.64937 & 36.64131 & 256.187 & 16 & 28 & 5 & 38 \\
\cline { 2 - 10 } & 420 & 1371 & 51.08242 & 36.98308 & 240.592 & 12 & 35 & 9 & 36 \\
\hline \multirow{5}{*}{ Non Touching } & 449 & 797 & 55.5688 & 34.03423 & 141.389 & 8 & 25 & 15 & 17 \\
\cline { 2 - 10 } & 489 & 1155 & 42.61806 & 36.27504 & 181.79 & 7 & 24 & 2 & 29 \\
\cline { 2 - 10 } & 285 & 847 & 46.49164 & 30.16958 & 156.663 & 26 & 5 & 2 & 28 \\
\cline { 2 - 10 } & 365 & 984 & 47.84766 & 31.93361 & 191.903 & 19 & 11 & 3 & 27 \\
\cline { 2 - 9 } & 348 & 885 & 50.96929 & 39.18898 & 173.741 & 23 & 12 & 2 & 30 \\
\cline { 2 - 9 } & 393 & 662 & 49.02589 & 29.05865 & 123.905 & 12 & 21 & 19 & 16 \\
\hline
\end{tabular}

Table 1 Sample of feature set
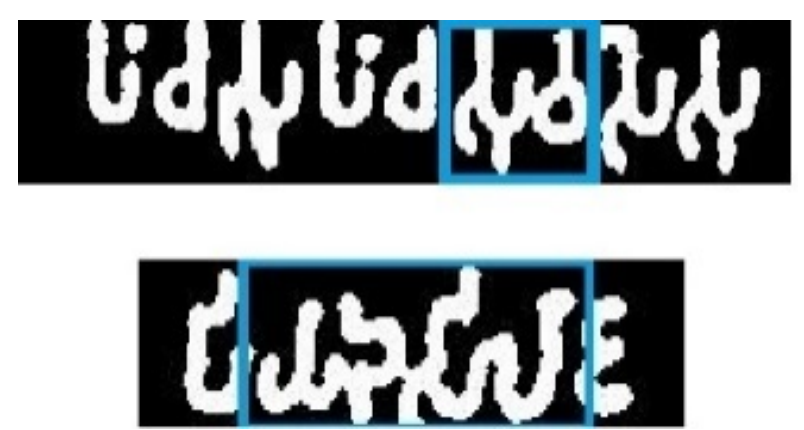

Fig. 3 Sample images of touching characters

We analyzed image set of 8014 (of which 963 are touching characters) segmented characters obtained from our previously developed algorithm for segmentation of Brahmi characters (Nagane and Mali (2020)).

\subsection{Segmentation of touching characters}

Once the touching characters are identified, they are segmented further into single characters. The touching characters may have two or more characters included. In our image set, the number of images containing two touching characters is the most. Three and four touching characters are also noticed considerably, whereas more number of touching characters are sparsely observed (Fig. 4). Table 2 describes this in detail. The pseudo-code for identification of the toughing characters is provided below: 


\section{Pseudo-code for identifying touching characters}

for $\mathrm{i}=1$ to $\mathrm{n} / / \mathrm{n}$ is number of images in a training directory read image;

//generate feature vector FD containing different features

TrainFD = $[\mathrm{f} 1, \mathrm{f} 2, \ldots, \mathrm{f} 9]$; endfor;

for $\mathrm{j}=1$ to $\mathrm{m} / / \mathrm{m}$ is number of images in a testing directory read image;

//generate feature vector FD containing different features $\mathrm{TestFD}=[\mathrm{f} 1, \mathrm{f} 2, \ldots, \mathrm{f} 9]$; endfor;

train the model using TrainFD ensembled classification technique test the trained model to predict the lables on TestFD

\begin{tabular}{|l|l|l|}
\hline Number of touching characters & Frequency & Percentage (\%) \\
\hline 2 & 743 & 9.27 \\
\hline 3 & 172 & 2.14 \\
\hline 4 & 41 & 0.51 \\
\hline 5 & 6 & 0.07 \\
\hline 6 & NIL & 0 \\
\hline 7 & 1 & 0.01 \\
\hline 8 or more & NIL & 0 \\
\hline Total & 963 & 12 \\
\hline
\end{tabular}

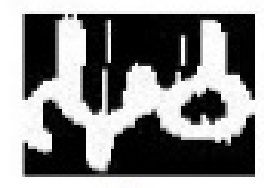

a

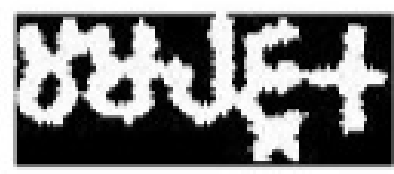

d

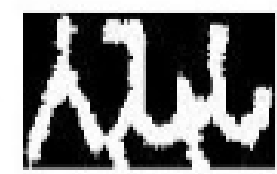

b

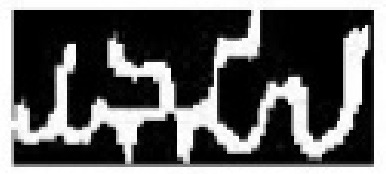

c

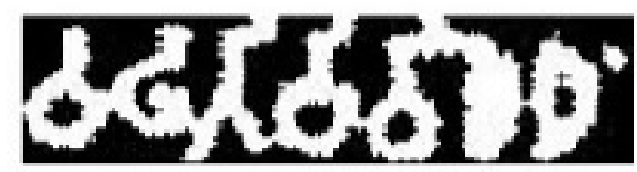

e

Fig. 4 Touching characters (a) two-touching characters; (b) three-touching characters; (c) four-touching characters; (d) five-touching characters; (e) seven-touching characters

To find correct segmentation points, initially we performed pre-processing on the image of touching characters. Morphological operations are executed in a sequence: closing, erosion, and thinning. For noise removal, median filter is applied after the 'close operation'. In the next step, we calculated the column-wise sum of foreground pixels. Further, we selected the columns as possible segmentation point (PSP) which has sum less than or equal to one. Then the final segmentation points (FSPs) were identified. For this, initially 7051 non-touching characters were analyzed to find the maximum number of columns required to represent the single Brahmi characters and a threshold was calculated.

Consider the first column as a marker (M). Compute the distance of column of PSP from M one by one until the distance is less than the threshold. Now, when the distance between the M and column from PSP is greater than threshold, add this column to FSP and also mark it as M. Repeat this process until all the columns from PSP are 
checked. Now, FSP contains the correct segmentation points. So, using them we segment the touching characters successfully (Fig. 5). The pseudo-code for the segmentation of touching characters is given below:

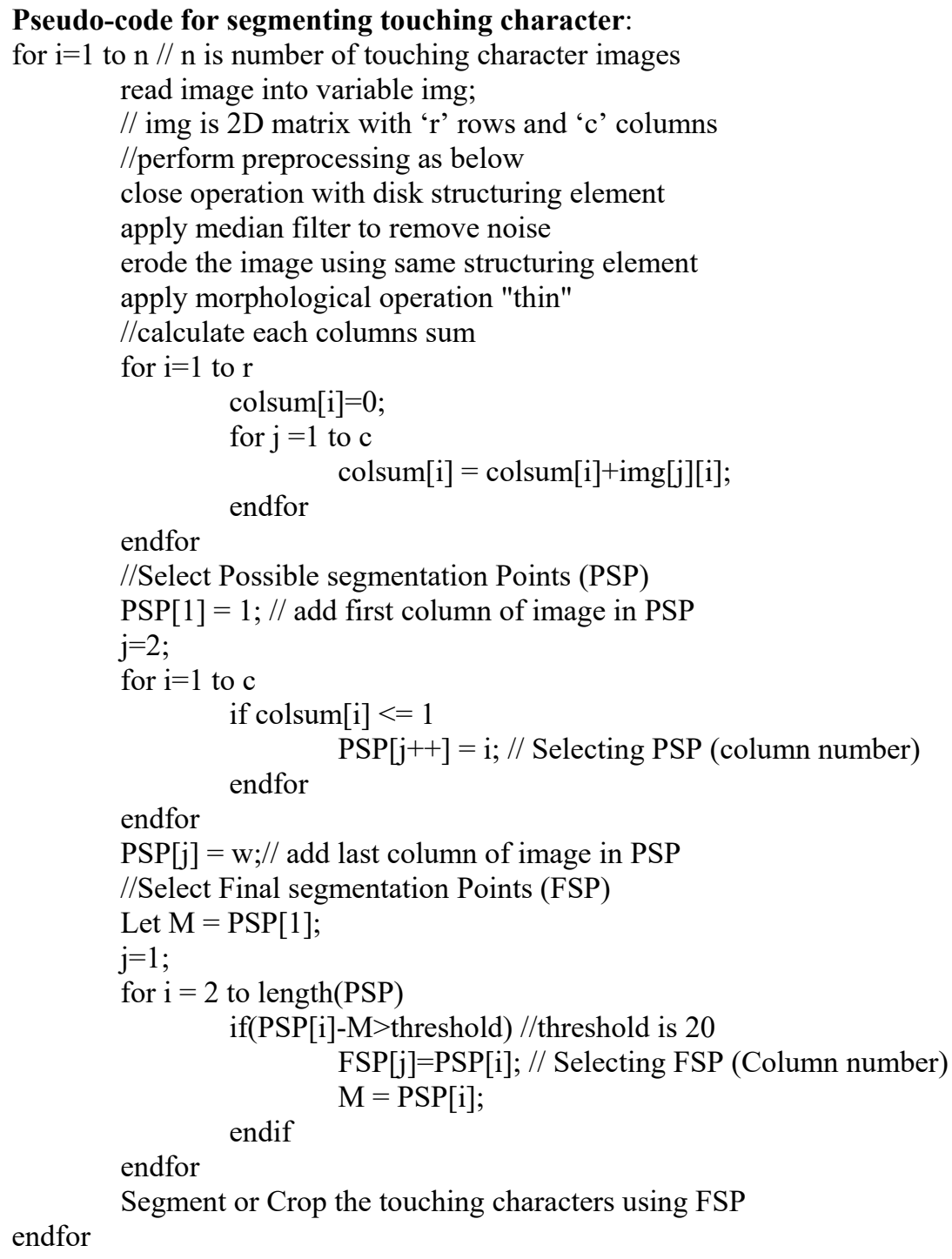

Segment or Crop the touching characters using FSP 


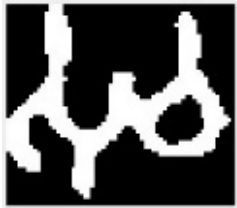

a

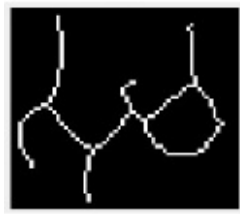

C

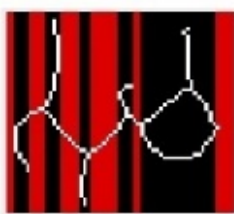

e

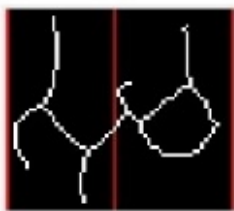

g

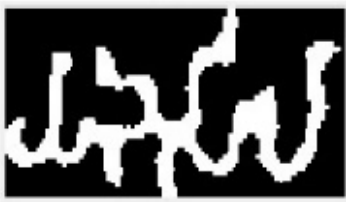

b

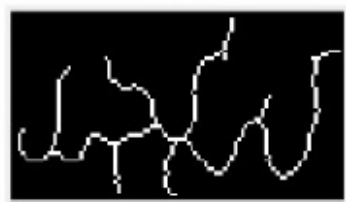

d

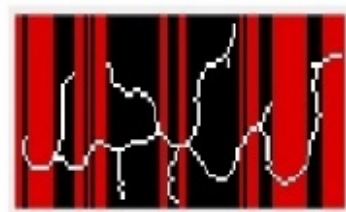

f

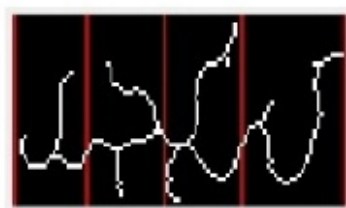

$\mathrm{h}$

Fig. 5 Step-by-step segmentation of touching characters (a, b) touching characters; (c, d) images after the preprocessing; (e, f) selected PSPs; $(\mathrm{g}, \mathrm{h})$ selected FSPs

\section{Results}

The proposed method for identifying and segmentation of touching characters is implemented in two steps. Initially, we implemented the algorithm for identification of touching characters on the set of 8014 images (containing touching 963 touching characters). The algorithm identified 974 images of touching characters, out of which 963 images are identified correctly. Here, the recognition rate of touching characters was $100 \%$. However, 11 extra single characters were identified as touching characters (Table 3). Furthermore, we implemented other classifiers to identify the touching characters which are support vector machine (SVM), Knearest neighbour $(\mathrm{KNN})$, and $\mathrm{K}$-means.

\begin{tabular}{|l|l|l|l|l|l|}
\hline Classification methods & $\begin{array}{l}\text { Total number } \\
\text { of images }\end{array}$ & $\begin{array}{l}\text { Number of single } \\
\text { characters }\end{array}$ & $\begin{array}{l}\text { Number of } \\
\text { touching characters }\end{array}$ & $\begin{array}{l}\text { Number of } \\
\text { touching characters } \\
\text { identified correctly }\end{array}$ & $\begin{array}{l}\text { Percentage of } \\
\text { identification (\%) }\end{array}$ \\
\hline Ensembled & 8014 & 7051 & 963 & 963 & 100 \\
\hline SVM & 8014 & 7051 & 963 & 819 & 85 \\
\hline KNN & 8014 & 7051 & 963 & 694 & 72 \\
\hline K-means & 8014 & 7051 & 963 & 193 & 20 \\
\hline
\end{tabular}

In the second phase, we implemented the algorithm for segmenting the touching characters on 963 images identified in previous step. These 963 images of touching characters contain 2203 individual characters. Total 955 images segmented successfully and resulted into correctly segmented 2187 individual characters. We achieved $99.16 \%$ of accuracy in segmentation of touching characters (Table 4 ). 


\begin{tabular}{|l|l|l|l|l|}
\hline $\begin{array}{l}\text { Total number of images of } \\
\text { touching characters }\end{array}$ & $\begin{array}{l}\text { Number of individual } \\
\text { characters in all images }\end{array}$ & $\begin{array}{l}\text { Correctly } \\
\text { segmented images }\end{array}$ & $\begin{array}{l}\text { Correctly segmented } \\
\text { individual characters }\end{array}$ & $\begin{array}{l}\text { Percentage of } \\
\text { identification (\%) }\end{array}$ \\
\hline 963 & 2203 & 955 & 2187 & 99.16 \\
\hline
\end{tabular}

Table 4 Results of touching character segmentation

Comparative analysis of various techniques used for the identification or segmentation or both is presented in Table 5. Methods compared are used on different scripts as no specific work in Brahmi script has been done so far.

\begin{tabular}{|c|c|c|c|c|}
\hline Reference & Language/Script & Technique & Dataset Size & Result \\
\hline Ullah et al. (2019) & Arabic & $\begin{array}{l}\text { Overlapping Set Theory and } \\
\text { Contour Tracing }\end{array}$ & 220 word images & $97.27 \%$ \\
\hline $\begin{array}{l}\text { Farulla and Murru } \\
(2017)\end{array}$ & Latin & Fuzzy logic & 1000 character images & $\begin{array}{l}\text { Without Noise: } 96.1 \% \\
\text { With Noise: } 88.9 \% \\
\text { (Max): }\end{array}$ \\
\hline Ali and Suresha (2019) & Arabic & $\begin{array}{l}\text { Characters geometry and shape- } \\
\text { based technique }\end{array}$ & $\begin{array}{l}13311 \text { character } \\
\text { images }\end{array}$ & $92.5 \%$ \\
\hline $\begin{array}{l}\text { Garain and Chaudhuri } \\
(2002)\end{array}$ & $\begin{array}{l}\text { Devnagari and } \\
\text { Bangla }\end{array}$ & $\begin{array}{l}\text { Fuzzy } \\
\text { Multifactorial Analysis }\end{array}$ & $\begin{array}{l}\text { Devnagari: } 11577 \\
\text { character images } \\
\text { Bangla: } 16714 \\
\text { character images }\end{array}$ & $\begin{array}{l}\text { Devnagari: } 57.48 \% \\
\text { Bangla: } 53.65 \%\end{array}$ \\
\hline $\begin{array}{l}\text { Deshmukh and Kolhe } \\
\text { (2019) }\end{array}$ & Modi & $\begin{array}{l}\text { Hybrid: Text zoning and column- } \\
\text { wise background pixel density } \\
\text { exploration }\end{array}$ & 1812 character images & $88.96 \%$ \\
\hline Roy et al. (2009) & Latin & $\begin{array}{l}\text { Dynamic programming using } \\
\text { primitive segments }\end{array}$ & 1790 character images & $90.39 \%$ \\
\hline Saba et al. (2011) & Latin & $\begin{array}{l}\text { Neural Confidence: Geometric } \\
\text { feature analysis and use of ANN }\end{array}$ & 2936 character images & $86.44 \%$ \\
\hline Proposed Method & Brahmi & $\begin{array}{l}\text { Connectivity based and sized based } \\
\text { analysis }\end{array}$ & 8014 character images & $\begin{array}{l}\text { Identification: } 100 \% \\
\text { Segmentation: } \\
99.16 \%\end{array}$ \\
\hline
\end{tabular}

Table 5 Comparison of results from various segmentation techniques.

\section{Conclusion}

In this paper, we have presented the method to identify and segment the touching characters from digitalized Brahmi script documents. The proposed method is implemented on already segmented images of characters using the earlier algorithm. The proposed methodology correctly identifies the touching characters and segments them into individual characters. The results are significant as we have achieved $100 \%$ accuracy in identification of touching characters using ensembled classification technique, whereas, the other techniques (SVM, KNN and K-means) have comparatively lower rate of touching character identification. Using the proposed method, we have achieved $99.16 \%$ of accuracy for segmentation of touching characters.

However, in the first phase, though we have identified all the toughing characters successfully, single characters were also identified as touching characters. Dynamic computation of threshold may help in addressing the problem. In case of segmentation of touching characters, there were characters that were not segmented accurately. This problem can be considered as over segmentation of the characters. So, there is a scope to work further on these problems and rectify them.

\section{Acknowledgments}

The authors would like to thank Dr. C. H. Patil for his academic support and valuable inputs in the research.

\section{References}

[1] Ali, A. A. A.; Suresha, M. (2019). An Efficient Character Segmentation Algorithm for Recognition of Arabic Handwritten Script. 2019 International Conference on Data Science and Communication (IconDSC), Bangalore, India, pp. 1-6, doi: 10.1109/IconDSC.2019.8817037.

[2] Burra, S.; Patel, A.; Bhagvati, C.; Negi, A. (2018). Improved Symbol Segmentation for TELUGU Optical Character Recognition. In: Abraham, A.; Muhuri, P.; Muda, A.; Gandhi, N. (Eds) Intelligent Systems Design and Applications. ISDA 2017. Advances in Intelligent Systems and Computing, vol. 736. Springer, Cham. https://doi.org/10.1007/978-3-319-76348-4 48

[3] Deshmukh, M. S.; Kolhe, S. R. (2019). A Hybrid Character Segmentation Approach for Cursive Unconstrained Handwritten Historical Modi Script Documents (February 23, 2019). Proceedings of International Conference on Sustainable Computing in Science, Technology and Management (SUSCOM), Amity University Rajasthan, Jaipur - India, February 26-28, Available at SSRN: https://ssrn.com/abstract=3356213 or http://dx.doi.org/10.2139/ssrn.3356213

[4] Farulla, G. A.; Murru, N.; Rossini, R. (2017). A fuzzy approach to segment touching characters. Expert Systems with Applications, 88, pp. 1-13.

[5] Garain, U.; Chaudhuri, B. B. (2002). Segmentation of touching characters in printed Devnagari and Bangla scripts using fuzzy multifactorial analysis. IEEE Transactions on Systems, Man, Cybernet Ics Part C, 32, pp. 449-459. doi:10.1109/TSMCC.2002.807272. 
[6] Garain, U.; Das, A.; Doermann, D. S.; Oard, D. D. (2012). Leveraging Statistical Transliteration for Dictionary-Based EnglishBengali CLIR of OCR'd Text. Proceedings of COLING 2012: Posters, pp. 339-348.

[7] Hamad, K.A.; Kaya, M. (2016). A Detailed Analysis of Optical Character Recognition Technology. International Journal of Applied Mathematics and Computer Science, 4(Special Issue), pp. 244-249.

[8] Jain, S. (2017). Optical Character Recognition System for Multilanguage Script Recognition. International Journal of Recent Research Aspects, 4, pp. 153-159.

[9] Jha, V.; Parvathy, K. (2019). Braille Transliteration of Hindi Handwritten Texts Using Machine Learning for Character Recognition. International Journal of Science and Technology Research, 8, pp. 1188-1193.

[10] Karthick, K.; Ravindrakumar, K.B.; Francis, R.; Ilankannan, S. (2019). Steps Involved in Text Recognition and Recent Research in OCR; A Study. International Journal of Recent Technology and Engineering, 8, pp. 3095-3100.

[11] Liu, H.; Zhang, L. (2019). Advancing Ensemble Learning Performance through data transformation and classifiers fusion in granular computing context. Expert Systems with Applications, 131, pp. 20-29. doi:10.1016/j.eswa.2019.04.051

[12] Mittal, R.; Garg, A. (2020). Text extraction using OCR: A Systematic Review. 2020 Second International Conference on Inventive Research in Computing Applications (ICIRCA), Coimbatore, India pp. 357-362, doi: 10.1109/ICIRCA48905.2020.9183326.

[13] Nagane, A. S.; Mali, S. M. (2020). Segmentation of Characters from Degraded Brahmi Script Images. In: Iyer, B., Rajurkar, A., Gudivada, V. (Eds). Applied Computer Vision and Image Processing. Advances in Intelligent Systems and Computing, vol. 1155. Springer, Singapore. https://doi.org/10.1007/978-981-15-4029-5_33

[14] Ojha, P. G. H. (1971). Bharatiya Prachin Lipimala: The Palaeography of India, Munshiram Manoharlal, New Delhi.

[15] Patil, C. H.; Mali, S. M. (2015). Segmentation of Isolated Handwritten Marathi Words. IJCA Proceedings of National Conference on Digital Image Signal Processing DISP. pp. 21-26.

[16] Peng, G.; Yu, P.; Li, H.; Li, H.; Zhu, X. (2017). A character segmentation algorithm for the palm leaf manuscripts, 2017 2nd IEEE International Conference on Computational Intelligence and Applications (ICCIA), Beijing, pp. 354-358, doi: 10.1109/CIAPP.2017.8167238.

[17] Rakshit, P.; Halder, C.; Ghosh, S.; Roy, K. (2018). Line, Word, and Character Segmentation from Bangla Handwritten Text-A Precursor Toward Bangla HOCR. In: Chaki, R.; Cortesi, A.; Saeed, K.; Chaki, N. (Eds). Advanced Computing and Systems for Security. Advances in Intelligent Systems and Computing, vol. 666. Springer, Singapore. https://doi.org/10.1007/978-981-10-81804_7

[18] Ramalingam, K.; Bhojan, R. (2018). Identification of Broken Characters in Degraded Documents. International Journal of Intelligent Engineering and Systems, 11, pp. 130-137.

[19] Rehman, A.; Saba, T. (2011). Performance analysis of character segmentation approach for cursive script recognition on benchmark database. Digital Signal Processing, 21, pp. 486-490.

[20] Roy, P. P.; Pal, U.; Lladós, J.; Delalandre, M. (2009). Multi-Oriented and Multi-Sized Touching Character Segmentation Using Dynamic Programming. 2009 10th International Conference on Document Analysis and Recognition, Barcelona, pp. 11-15, doi: 10.1109/ICDAR.2009.124.

[21] Saba, T.; Rehman, A.; Elarbi-Boudihir, M. (2014). Methods and strategies on off-line cursive touched characters segmentation: a directional review. Artificial Intelligence Review, 42, pp. 1047-1066. https://doi.org/10.1007/s10462-011-9271-5

[22] Saba, T.; Rehman, A.; Sulong, G. (2011). Cursive Script Segmentation with Neural Confidence. International Journal of Innovative Computing Information and Control, 7, pp. 4955-4964.

[23] Salomon, R. (1998). Indian Epigraphy: A Guide to the Study of Inscriptions in Sanskrit, Prakrit, and the Other Indo-Aryan Languages. Oxford University Press, New York Oxford.

[24] Sen, S.; Chowdhury, S.; Mitra, M.; Schwenker, F.; Sarkar, R.; Roy, K. (2018). A novel segmentation technique for online handwritten Bangla words. Pattern Recognition Letters [in press]. https://doi.org/10.1016/j.patrec.2018.02.008

[25] Ullah, I.; Azmi, M. S.; Desa, M. I.; Alomari, Y. M. (2019). Segmentation of Touching Arabic Characters in Handwritten Documents by Overlapping Set Theory and Contour Tracing. International Journal of Advanced Computer Science Applications, 10, $\quad$ pp. 155-160. URL: https://en.wikipedia.org/wiki/Estampage\#: :text=From\%20Wikipedia\%2C\%20the\%20free\%20encyclopedia,paper\%20for\%20a\%2 0clearer\%20read [accessed on October 4, 2020]

[26] Zho, H.; Zhu, G.; Peng, Y. (2016). A RMB optical character recognition system using FPGA, 2016 IEEE International Conference on Signal and Image Processing (ICSIP), Beijing, pp. 539-542, doi: 10.1109/SIPROCESS.2016.7888320.

Authors Profile

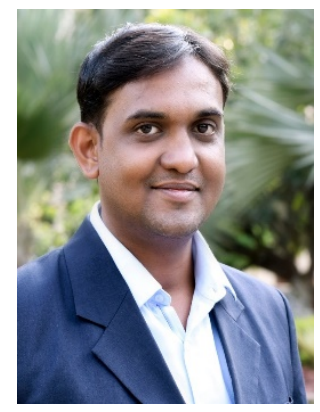

Mr. Aniket Suresh Nagane is currently working as Assistant Professor at Department of Computer Science, MAEER's MIT Arts, Commerce and Science College, Alandi(D), Pune. He has completed his Masters' degree in Computer Science in 2009 and qualified NET examination. He is currently pursuing his Ph.D. from Savitriabai Phule Pune University, Pune under the guidance of Dr. S. M. Mali. He has published 5 research papers in national and international journals and has more than 12 years of experience in teaching. 
Dr. Shankar Maruti Mali is currently working as Professor at School of Computer Science, MIT World Peace University. He is also the Coordinator of IQAC at MITWPU. He completed his PhD degree from Solapur University in 2011 and qualified SET in Computer Science in 2004. He is currently guiding $6 \mathrm{PhD}$ scholars at Savitribai Phule Pune University and MIT World Peace University. His research areas include Digital image processing, Pattern recognition, Natural language processing and Networking. He has published 30 research papers in national and international journals. He has more than 22 years of experience in teaching. He is a member of Research and Recognition Committee at MIT-WPU and also a member of board of studies at different autonomous colleges in Pune. Previously, he has worked as Head of Department, Vice-Principal, BOS Chairman, Selection Committee Member and LIC Committee Member. 